\title{
Varicocelectomy and infertility
}

Jerzy B. Gajewski

Department of Urology, Dalhousie University, Halifax, Canada

There is no doubt that presence of varicocele contributes to testicular atrophy and decreased fertility in men [1]. There is also evidence that varicocelectomy improved quality of sperm and improve chances of pregnancy although some randomized controlled study were less impressive [2]. Obviously not every man with a significant varicocele has fertility issues or will benefit from varicocelectomy, but the odds favours performing surgery in these patients. It is still unclear through which mechanism varicocelectomy improves fertility. The possible options include decrease in hydrostatic pressure and venous reflex to the testis. There are also data indicating normalization of the testicular temperature and reduction in venous volume stabilize intratesticular testosterone metabolism (testicular proteins exhibit a reduced thermal stability) and improve spermatogenesis [3]. The present study of Bryniarski et al. [4] showed significant benefit of varicocelectomy for testicular growth with the initial hypotrophic testis using laparoscopic approach and confirming previous results in literature. The main advantage of this technique is optical magnification allowing preservation of lymphatics and testicular artery. The authors did not reported any complications with this technique, but it has to be remembered that although it is so called "minimal invasive", it has its own complications and consequences. It increases cost of instrumentation and has infrequent but more serious complication including injury to the bowel or other organs, perito- nitis or air embolism [5]. For that reason, microsurgical subinguinal technique which combines optical magnification for better exposure with easy and simple access, gains popularity [6]. It is also associated with less postoperative pain.

It is still difficult to directly correlate improvement in testicular size to the improved sperm function or pregnancy rate. The present study would be more interesting if sperm quality and hormonal status was correlated to the grade of varicocele and tested after varicocelectomy. Indirectly, there were reports in literature, indicating that the sperm quality is inferior in a lot of patients with hypotrophic testis and with higher grade of varicocele. This improves after varicocelectomy [7]. The pregnancy rate increases only up to $50 \%$ after varicocelectomy, indicating that even with improved testicular size and/or sperm quality the pregnancy rate are far from optimal. It should be taken into consideration however that there are other factors influencing pregnancy rate equally on men and women site.

There is also a question of possible beneficial effect of varicocelectomy on preventing development of late onset of hypogonadal state [8]. Confirmation of that statement obviously will require lengthy longitudinal population study.

Varicocelectomy has its place in management of infertile men; however more studies are still required to fine tune evaluation, indication and technique of this intervention.

\section{References}

1. Pinto KJ, Kroovand RL, Jarow JP. Varicocele related testicular atrophy and its predictive effect upon fertility. J Urol. 1994; 152: 788-790.

2. Krause W, Muller HH, Schafer $\mathrm{H}$, Weidner W. Does treatment of varicocele improve male fertility? Results of the 'Deutsche Varikozelenstudie', a multicentre study of 14 collaborating centres. Andrologia. 2002; 34: 164-171.

3. Lue YH, Lasley BL, Laughlin LS, Swerdloff R, Hikim AP, Leung A, et al. Mild testicular hyperthermia induces profound transitional spermatogenic suppression through increased germ cell apoptosis in adult cynomolgus monkeys (Macaca fascicularis). J Androl. 2002; 23: 799-805.
4. Bryniarski P, Kaletka Z, Huk J, Fryczkowski M, Prokopowicz G, Życzkowski M, et al. Testicular volume and fertility potential in men operated due to varicocele and testicular hypotrophy in adolescence. CEJU. 2013; 66: 56-59.

5. Enquist E, Stein BS, Sigman M. Laparoscopic versus subinguinal varicocelectomy: a comparative study. Fertil Steril. 1994; 61: 1092-1096.

6. Cayan S, Kadioglu TC, Tefekli A, Kadioglu A, Tellaloglu S. Comparison of results and complications of high ligation surgery and microsurgical high inguinal varicocelectomy in the treatment of varicocele. Urology. 2000; 55: 750-754
7. Su L-M, Goldstein M, Schlegel PN. The effect of varicocelectomy on serum testosterone levels in infertile men with varicoceles. J Urol. 1995; 154: 1752-1755.

8. Srini VS and Veerachari SB. Does Varicocelectomy Improve Gonadal Function in Men with Hypogonadism and Infertility? Analysis of a Prospective Study. Int J Endocrinol. 2011; 2011: doi:10.1155/2011/916380.

\section{Correspondence} Prof. Jerzy B. Gajewski, MD, FRCSC jgajew@dal.ca 